\title{
Radiation Induced Exacerbation of Bullous Pemphigoid in Rectal Cancer
}

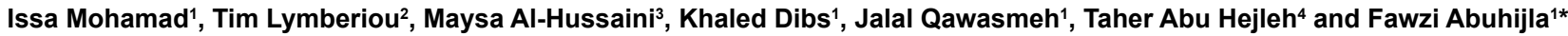

${ }^{1}$ Department of Radiation Oncology, King Hussein Cancer Center, Amman, Jordan

${ }^{2}$ Department of Radiation Oncology, CISSS Montérégie-Centre, Montreal, Canada

${ }^{3}$ Department of Pathology, King Hussein Cancer Center, Amman, Jordan

${ }^{4}$ Department of Internal Medicine, Medical Oncology, King Hussein Cancer Center, Amman, Jordan

\begin{abstract}
Neoadjuvant chemo-radiotherapy followed by surgery is the treatment of choice for locally advanced rectal cancer. We report a case of a 63-year-old woman with a history of bullous pemphigoid (BP) in remission, presenting with locally advanced rectal adenocarcinoma, which developed severe exacerbation of BP during neoadjuvant concurrent chemotherapy and radiation therapy. A dramatic response in the BP flare up was achieved with a oneweek treatment break in radiation and chemotherapy in addition to administering corticosteroids (CS).
\end{abstract}

Keywords: Radiation; Radiotherapy; Bullous pemphigoid

\section{Introduction}

Bullous pemphigoid (BP) is an autoimmune sub-epidermal blistering disease caused by activation of the complement system and an inflammatory-mediated process at the level of the skin basement membrane [1]. Radiotherapy (RT) has shown improved outcomes in preoperative settings in rectal cancer [2,3]. Skin damage by entities such RT have been shown to trigger these dermatologic processes, both during and after RT [4-6], leading to severe local exacerbation of BP. Prompt discontinuation of RT and administration of corticosteroids (CS) potentially result in recuperation of the BP flare up [1], possibly allowing for resumption of RT especially in cancer cases treated with curative intent. Herein, we present a case of radiation induced BP exacerbation in a patient with rectal cancer undergoing neoadjuvant concurrent chemotherapy and radiation therapy.

\section{Case Report}

A 63-year-old woman with a history of BP in remission presented with pelvic discomfort, constipation, and rectal bleeding. She had comorbidities including hypertension, coronary artery disease, and type II diabetes mellitus. Digital rectal examination followed by colonoscopy revealed a large rectal mass at $7 \mathrm{~cm}$ from the anal margin, extending $5 \mathrm{~cm}$ superiorly. Biopsy from the rectal mass was consistent with moderately differentiated adenocarcinoma. MRI pelvis showed the rectal mass with peri-rectal fat involvement and pelvic lymphadenopathy. The stage assigned to the cancer was cT3N2M0. The case was discussed in the gastrointestinal multidisciplinary meeting, and the recommendation was to treat with neoadjuvant radiotherapy with concurrent capecitabine. The patient was planned to receive external beam RT for a total dose of 45 Gy over 25 fractions to the pelvis, covering the peri-rectal, internal iliac, obturator, and pre-sacral nodal regions, using a 4 field-box technique and 15 Mega Volt (MV) photon energy (Figures 1a-1d).

During the third week of RT (at 25.2 Gy), the patient suffered from painful blistering of the skin confined to the field of radiation (Figure 2a and $2 \mathrm{~b}$ ).. She was treated with systemic prednisolone at a dose of $1 \mathrm{mg} /$ $\mathrm{kg}$ daily for 14 days, and a combination cream of topical betamethasone and fusidic acid was applied as a thin layer over the affected area twice daily. At $32.4 \mathrm{~Gy}$, skin blisters had progressed and the patient required one-week RT and chemotherapy treatment break, after which the skin dermatosis improved by approximately $50 \%$ (Figure $2 \mathrm{c}$ and $2 \mathrm{~d}$ ).

Biopsy of the skin blisters showed sub-epidermal bullous lesion, consistent with bullous pemphigoid exacerbation (Figures $3 \mathrm{a}$ and $3 \mathrm{~b}$ ).
The patient refused to continue treatment at this time. Re-imaging at 6 weeks post 32.4 Gy by 18 fractions with MRI showed small residual tumor at the mid/upper rectum and small regional lymph nodes. The overall picture was consistent with partial response without distant metastases.

Subsequently, the patient underwent a low anterior resection (LAR). Pathology showed partial response to the neoadjuvant treatment, ypT2N0 moderately differentiated adenocarcinoma (Figure 4), maximal tumor dimension of $3 \mathrm{~cm}$, negative surgical margins, with 15 lymph nodes were negative for metastasis. She was referred to medical oncology for adjuvant chemotherapy; however, this was

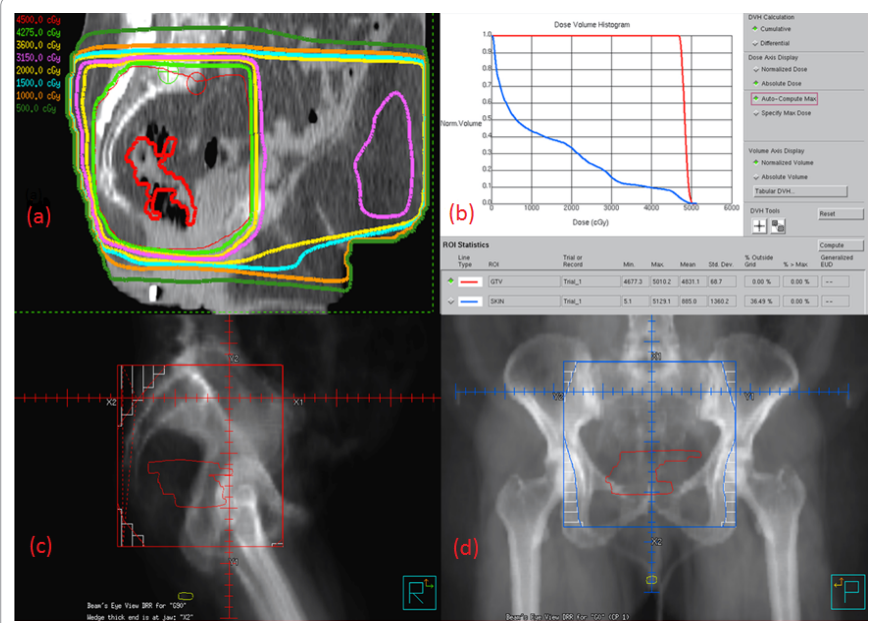

Figure 1a and 1b: Pelvic CT simulation image showing radiotherapy isodose distribution in the pelvic area, radiatherapy skin dose on the dose volume histogram and Figure 1c and 1d: radiotherapy DRR for right and posterior radiation fields.

${ }^{*}$ Corresponding author: Fawzi Abuhijla, King Hussein Cancer Center, PO Box 1269, Amman 11941, Jordan, Tel: +962796500577; E-mail: fhijle@khcc.jo

Received February 14, 2018; Accepted March 29, 2018; Published April 05, 2018

Citation: Mohamad I, Lymberiou T, Al-Hussaini M, Dibs K, Qawasmeh J, et al. (2018) Radiation Induced Exacerbation of Bullous Pemphigoid in Rectal Cancer. J Oncol Med \& Pract 3: 119. doi:10.4172/2576-3857.1000119

Copyright: @ 2018 Mohamad I, et al. This is an open-access article distributed under the terms of the Creative Commons Attribution License, which permits unrestricted use, distribution, and reproduction in any medium, provided the original author and source are credited. 


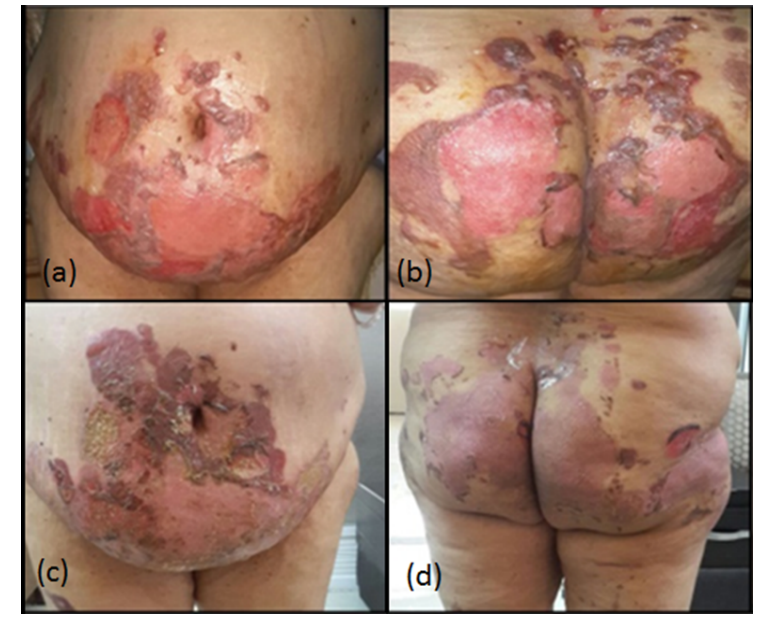

Figure $\mathbf{2 a}$ and $\mathbf{2 b}$ : Blisters of the anterior abdominal wall and skin over the buttocks area after 25.2 Gy. Figure 2c and 2d: Healing of skin blisters after one week break.

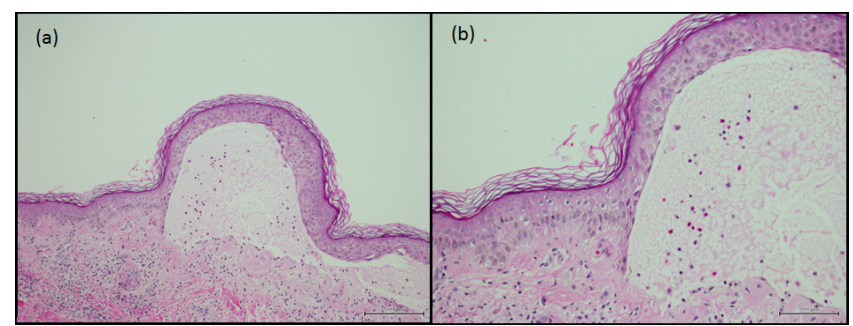

Figure 3a and 3b: Low and medium power view of the skin biopsy showing subepidermal bullous formation. The bullous contains inflammatory cells including eosinophils. Perivascular inflammatory cell infiltrate was also seen, including many eosinophils. (H\&E, X10 and 20 , respectively).

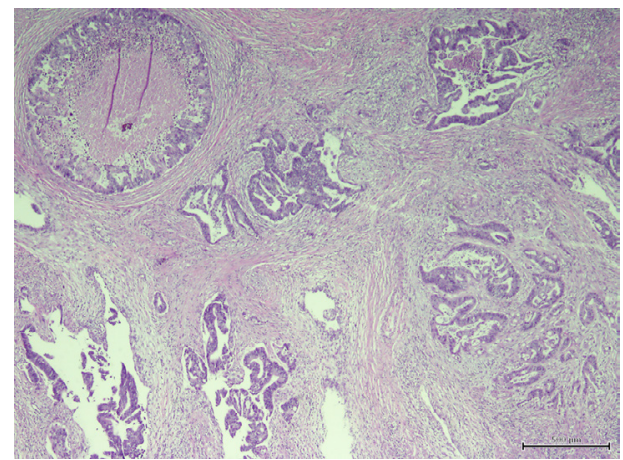

Figure 4: Residual viable colorectal carcinoma post neoadjuvant radiotherapy Most of the tumor is viable with minimal post-therapy changes. (H\&E, X4).

refused by the patient. Six months following the surgery, the patient was still free of cancer recurrence.

\section{Discussion}

In this report, we present a case of severe flare up of previously wellcontrolled $\mathrm{BP}$ in a patient receiving neoadjuvant concurrent chemotherapy and radiation therapy for locally advanced rectal cancer. To the authors' knowledge, this is the first case of local BP exacerbation induced by radiation in a patient with rectal carcinoma as most of the cases have been reported in breast cancer patients receiving radiation [5,7].
In this case, the skin area involved by the BP exacerbation was confined to the radiation field likely indicating a causal linkage to RT which could have been aggravated by the concomitant use of capecitabine. In the systematic review by Mul [5] where most of the $\mathrm{BP}$ cases had primary breast cancer, the pelvic radiation cases resulted in generalized exacerbation of the BP disease; which is inconsistent with the localized toxicity noticed in our case. The generalized BP exacerbation in the pelvic radiation cases was also noticed by Nguyen [7]. The generalized toxicity probably reflects an immune mediated mechanism of toxicity resulting from the skin injury caused by radiation $[5,7]$.

The BP flare up in our case occurred at 25.2 Gy. As per Mul [5] most of the BP exacerbations occurred after a median radiation dose of $50 \mathrm{~Gy}$ with only 4 patients developing flare up at 20 and $32 \mathrm{~Gy}$. This is consistent with the finding by Nguyen [7], where only 2 patients had exacerbations at a radiation dose of $20 \mathrm{~Gy}$ and $46 \mathrm{~Gy}$. Whether the primary site of the tumor being in the breast versus other sites affects the time of onset of the skin toxicity is unclear. Additionally, the hormonal treatment that is commonly used in breast cancers might also have an implication on the development of BP exacerbation in breast radiation, possibly explaining the late dermatologic toxicities seen in breast cancer more often than other primary cancers. Our patient had significant remission of the BP exacerbation after 14 days of prednisone treatment and complete cessation of radiotherapy and capecitabine. Continuing radiation was suggested as long as the dermatologic toxicity is not prohibitive [5].

\section{Conclusion}

In conclusion, BP exacerbation is common in patients receiving radiation. However, this toxicity is manageable with CS and is not an absolute contraindication to the continuation or resumption of radiotherapy after improvement. Potential interventions to overcome this challenging side effect include early introduction of CS treatment after starting radiation, using intensity modulated radiotherapy (IMRT) or volumetric modulated arc therapy (VMAT) instead of three dimensional conformal radiotherapy (3D-CRT), choosing chemotherapies with less dermatologic toxicities, as well as the integration of the dermatology service in the multidisciplinary treating team.

\section{Funding}

None

\section{Conflict of Interest}

Authors declare no conflict of interest.

All authors contributed in manuscript writing and reviewing.

Formal consent obtained from patient.

\section{References}

1. Lo Schiavo A, Ruocco E, Brancaccio G, Caccavale S, Ruocco V, et al. (2013) Bullous pemphigoid: Etiology, pathogenesis, and inducing factors: Facts and controversies. Clin Dermatol 31: 391-399.

2. Swedish Rectal Cancer Trial, Cedermark B, Dahlberg M, Glimelius B, Pahlman $\mathrm{L}$, et al. (1997) Improved survival with preoperative radiotherapy in resectable rectal cancer. N Engl J Med 336: 980-987.

3. Sauer R, Liersch T, Merkel S, Fietkau R, Hohenberger W, et al. (2004) Preoperative versus postoperative chemoradiotherapy for rectal cancer. N Engl J Med 351: 1731-1740.

4. Frederich M (1989) Hospice health care: Management of a case of bullous pemphigoid utilizing the hospice interdisciplinary team. Hosp J 5: 79-83.

5. Ho C, Tong KM, Ramsden K, Ionescu DN, Laskin J, et al. (2015) Histologic 
Citation: Mohamad I, Lymberiou T, Al-Hussaini M, Dibs K, Qawasmeh J, et al. (2018) Radiation Induced Exacerbation of Bullous Pemphigoid in Rectal Cancer. J Oncol Med \& Pract 3: 119. doi:10.4172/2576-3857.1000119

Page 3 of 3

classification of non-small-cell lung cancer over time: Reducing the rates of not-otherwise-specified. Curr Oncol 22: e164-170.

6. Parikh SK, Ravi A, Kuo DY, Nori D (2001) Bullous pemphigoid masquerading as acute radiation dermatitis: Case report. Eur J Gynaecol Oncol 22: 322-324.

7. Nguyen T, Kwan JM, Ahmed AR (2014) Relationship between radiation therapy and bullous pemphigoid. Dermatology 229: 88-96. 